Poincaré-Lie algebra and relativistic phase space

This article has been downloaded from IOPscience. Please scroll down to see the full text article.

2000 J. Phys. A: Math. Gen. 334015

(http://iopscience.iop.org/0305-4470/33/22/306)

View the table of contents for this issue, or go to the journal homepage for more

Download details:

IP Address: 200.130.19.138

The article was downloaded on 24/07/2013 at 20:53

Please note that terms and conditions apply. 


\title{
Poincaré-Lie algebra and relativistic phase space
}

\author{
M C B Andrade†, A E Santana $\uparrow$ and J D M Vianna $\uparrow$ \\ $\dagger$ Instituto de Física, Universidade Federal da Bahia, Campus de Ondina, 40210-340, Salvador, \\ Bahia, Brazil \\ \$ Instituto de Física, Universidade de Brasília, 70910-900, Brasília-DF, Brazil
}

Received 25 May 1999, in final form 21 March 2000

\begin{abstract}
In this work we investigate representations of the Poincaré group taking as the representation space the Hilbert space of thermofield dynamics, a real-time formalism developed in quantum field theory at finite temperature. We concentrate our study on those representations that give rise to the notion of phase space, with direct application in kinetic theory. As a result, we show an alternative way to derive a relativistic Boltzmann equation, based on the notion of a propagator defined in phase space. The quantum counterpart of the approach is discussed through the notion of the Wigner function.
\end{abstract}

\section{Introduction}

Thermal equilibrium properties of a quantum field can be analysed through the celebrated imaginary-time formalism, first proposed by Matsubara [1]. An algebraic counterpart of Matsubara's method is the so-called thermofield dynamics (TFD) [2,3], which is structurally based on the notion of vector space. In other words, operators in TFD are defined by a doubling of the degrees of freedom of the system, the tilde (or dual) conjugation rules. A Bogoliubov transformation then introduces thermal operators, keeping invariant the algebraic rules of the non-thermal operators, such that the thermal states are considered as condensed states.

Doubling procedures in thermal field theory have been recognized as a suitable amendment to treat temperature in a real-time formalism, providing, in addition, correct pertubative expansions (for a detailed account see [4]). In particular, in TFD, doubling of the degrees of freedom is carried out on a solid algebraic basis [5,6], and consequently it has been explored in different ways, as for instance, to define thermal coherent and squeezing states [7,8], or still in association with structures of Hopf algebras $[9,10]$. In the same context, Lie groups have been studied as for the case of $S U(2)$, furnishing spin models with interesting perturbative techniques [11]. Recently, the Galilei and Poincaré groups have been analysed [6,12], opening the possibility of dealing with kinetic theory from a group theoretical standpoint. This aspect is of interest in high-energy physics, where attempts to improve the kinetic theory have been undertaken in order to accommodate gauge symmetry $[13,14]$.

On the other hand, in Galilean hydrodynamics theory and diffusion processes, a variety of studies have been undertaken using second-quantization methods but applied to (nonrelativistic) Liouville and transport equations [15-17]. Here, such methods are generalized to relativistic situations, starting with the TFD-representation theory to study the PoincaréLie algebra. Then, the relativistic kinetic theory is introduced through a Hilbert space. In this realm of representation, we use the notion of a propagator in the phase space to derive a 
Boltzmann-like equation. The non-relativistic limit of such an approach is achieved and the equivalent quantum formalism is discussed via the concept of Wigner density.

This paper is organized as follows. In section 2 a résumé of TFD is presented focusing on algebraic aspects that will be explored in subsequent sections, as the concept of representation of Lie algebra based in the TFD Hilbert space. In section 3 representation of the Poincaré-Lie algebra is explored in such a context. As an application, a relativistic Boltzmann equation is derived in section 4, and the analysis of the non-relativistic limit is carried out. Quantum aspects of such a formalism are discussed in section 5, and in section 6 final remarks and conclusions are presented.

\section{TFD and Lie algebras}

In statistical mechanics, the canonical-Gibbs average of an operator $A$ is given by

$$
\langle A\rangle=\frac{1}{Z} \operatorname{Tr} \mathrm{e}^{-\beta H} A
$$

where $\beta=1 / k_{\mathrm{B}} T$, with $k_{\mathrm{B}}$ being the Boltzmann constant, $T$ the temperature, and $H$ the Hamiltonian. TFD was motived by the fact that equation (1) can be written as $[2,3]$

$$
\langle A\rangle=\langle 0(\beta)|A| 0(\beta)\rangle
$$

where the thermal state $|0(\beta)\rangle$ is a Hilbert space vector. An immediate advantage of such a procedure is that pertubative techniques can be developed in line with the zero-temperature formalism. Let us then see how this works in the case of bosons.

In order to accomplish the goal of writing equation (1) in the bilinear form given by equation (2), a doubling in the boson operators is defined in the following sense. Considering $a$ a bosonic operator, another bosonic operator, say $\tilde{a}$, is introduced such that the tilde and non-tilde variables fullfil the following algebra:

$$
\begin{aligned}
& {\left[a_{k}, a_{k^{\prime}}^{\dagger}\right]=\left[\tilde{a}_{k}, \tilde{a}_{k^{\prime}}^{\dagger}\right]=\delta_{k k^{\prime}}} \\
& {\left[a_{k}, \tilde{a}_{k^{\prime}}\right]=\left[a_{k}, \tilde{a}_{k^{\prime}}^{\dagger}\right]=0 .}
\end{aligned}
$$

This mapping of $a \rightarrow \tilde{a}$ gives rise to a doubling in the Hilbert space of the original system, $|\phi\rangle \rightarrow|\phi, \tilde{\phi}\rangle=|\phi\rangle \otimes|\tilde{\phi}\rangle$, characterized by

$$
\begin{aligned}
& a|\phi\rangle \rightarrow a|\phi, \tilde{\phi}\rangle=(a|\phi\rangle) \otimes|\tilde{\phi}\rangle \\
& \tilde{a}|\tilde{\phi}\rangle \rightarrow \tilde{a}|\phi, \tilde{\phi}\rangle=|\phi\rangle \otimes(\tilde{a}|\tilde{\phi}\rangle) \stackrel{\text { def }}{=}|\phi\rangle \otimes\left(\langle\phi| a^{\dagger}\right) .
\end{aligned}
$$

In this way, the state $|0(\beta)\rangle$ is defined by

$$
\begin{aligned}
|0(\beta)\rangle & =Z^{-1 / 2}(\beta) \sum_{n} \exp (-\beta n \omega / 2)|n, \tilde{n}\rangle \\
& =\left(1-\mathrm{e}^{\beta \omega}\right) \exp \left\{\exp \left[(-\beta \omega / 2) a^{\dagger} \tilde{a}^{\dagger}\right]\right\}|0, \tilde{0}\rangle
\end{aligned}
$$

satisfying $\langle 0(\beta) \mid O(\beta)\rangle=1$ (at this point we have suppressed the mode index, $k$, for simplicity).

For the particular case in which in equation (1) $A=n=a^{\dagger} a$, from equation (5) it results that

$$
\left.n=\langle 0(\beta)\rangle\left|a^{\dagger} a\right| 0(\beta)\right\rangle=\frac{1}{1-\mathrm{e}^{-\beta \epsilon}} .
$$

Therefore, in this context, the physical variables are described by non-tilde operators, whilst the tilde operators, up to now, play a role of auxiliary variables. A physical meaning for tilde variables, however, will be given in the next section when we analyse the TFD structure in connection with symmetry groups. 
With such results, a way to recover the density matrix formalism is to observe that equation (5) can be written as

$$
\begin{aligned}
|0(\beta)\rangle & =\rho^{1 / 2}|1\rangle \\
& =Z^{-1 / 2}(\beta) \sum_{n} \exp (-\beta n \omega / 2)|n, \tilde{n}\rangle
\end{aligned}
$$

where $\rho^{1 / 2}=Z^{-1 / 2}(\beta) \exp \left(-\beta \omega a^{\dagger} a / 2\right)$ and $|1\rangle=\sum_{n}|n, \tilde{n}\rangle$. Following this scheme, the notion of the square root of the density matrix $\rho^{1 / 2}$ (see also $[18,19]$ ) is used to define physical states even for non-equilibrium conditions [20,21].

The results derived above can be written in a very suggestive and general form via a Bogolubov transformation introduced by

$$
U(\beta)=\mathrm{e}^{-\mathrm{i} G}
$$

where $G=-\mathrm{i} \theta(\beta)\left(a^{\dagger} \tilde{a}^{\dagger}-a \tilde{a}\right)$, and $\theta(\beta)$ is defined via

$$
\begin{aligned}
& \cosh \theta(\beta)=\frac{1}{\left[1-\mathrm{e}^{-\beta \epsilon}\right]^{1 / 2}} \equiv u(\beta) \\
& \sinh \theta(\beta)=\frac{1}{\left[\mathrm{e}^{\beta \epsilon}-1\right]^{1 / 2}} \equiv v(\beta) .
\end{aligned}
$$

Using equation (7), the thermal state $|0(\beta)\rangle$ is given by $|0(\beta)\rangle=U(\beta)|0, \tilde{0}\rangle$. On the other hand, thermal operators are introduced by

$$
a(\beta)=U(\beta) a U^{\dagger}(\beta) \quad \text { and } \quad \tilde{a}(\beta)=U(\beta) \tilde{a} U^{\dagger}(\beta) .
$$

It is a simple exercise to show that $a(\beta)$ and $\tilde{a}(\beta)$ satisfy the same algebraic relation as those given in equations (3) and (4), such that $a(\beta)|0(\beta)\rangle=\tilde{a}(\beta)|0(\beta)\rangle=0$. That is, the thermal state $|0(\beta)\rangle$ is a vacuum for $a(\beta)$ and $\tilde{a}(\beta)$ (but not for $a$ and $\tilde{a}$ ). As a result, the thermal vacuum average of a non-thermal operator is equivalent to the Gibbs canonical average in statistical physics.

In order to treat infinite degrees of freedom, it is convenient to introduce a thermal doublet notation. That is, for an arbitrary operator $A$ we define

$$
\left(A^{a}\right)=\left(\begin{array}{c}
A(\beta) \\
\tilde{A}^{\dagger}(\beta)
\end{array}\right) \quad\left(A^{a+}\right)=\left(A^{\dagger}(\beta) \tilde{A}(\beta)\right) .
$$

Then, the algebraic rules for the thermal bosonic operators are written as $\left[a^{a}(\beta), a^{b+}(\beta)\right]=\delta^{a b}$ where $a, b=1,2$. The Bogoliubov transformation, equation (7), is therefore written as a $2 \times 2$ matrix,

$$
\mathcal{B}=\left(\begin{array}{cc}
u(\beta) & -v(\beta) \\
-v(\beta) & u(\beta)
\end{array}\right)
$$

such that equation (10) reads $a^{a}=\left(\mathcal{B}^{-1}\right)^{a b} a^{b}(\beta)$ and $a^{+a}=a^{b}(\beta) \mathcal{B}^{b a}$. Writing explicitly, we have

$$
a=u(\beta) a(\beta)+v(\beta) \tilde{a}^{\dagger}(\beta) \quad \text { and } \quad \tilde{a}=u(\beta) \tilde{a}(\beta)+v(\beta) a^{\dagger}(\beta) .
$$

As an example of a system with infinite modes, consider the free Klein-Gordon field. In this situation, the way to recover the usual results is through the definition of the following Lagrangian:

$$
\hat{L}=\frac{1}{2} \partial_{\mu} \phi(x) \partial^{\mu} \phi(x)-\frac{m^{2}}{2} \phi^{2}-\frac{1}{2} \partial_{\mu} \tilde{\phi}(x) \partial^{\mu} \tilde{\phi}(x)+\frac{m^{2}}{2} \tilde{\phi}(x)^{2} .
$$


The two-points Green function is then defined by

$$
\begin{aligned}
G\left(x-x^{\prime} ; \beta\right)^{a b} & =\langle 0(\beta)| T\left[\phi(x)^{a} \phi\left(x^{\prime}\right)^{b}|0(\beta)\rangle\right. \\
& =\frac{1}{(2 \pi)^{4}} \int \mathrm{d}^{4} k G(k ; \beta)^{a b} \mathrm{e}^{\mathrm{i} k\left(x-x^{\prime}\right)}
\end{aligned}
$$

where $G(k ; \beta)^{a b}=\mathcal{B}^{-1}\left(k_{o}\right) G_{o}(k)^{a b} \mathcal{B}\left(k_{o}\right)$, with

$$
\left(G_{o}(k)^{a b}\right)=\left(\begin{array}{cc}
\frac{1}{k^{2}-m^{2}+\mathrm{i} \epsilon} & 0 \\
0 & \frac{-1}{k^{2}-m^{2}-\mathrm{i} \epsilon}
\end{array}\right) .
$$

Using the definition of $\mathcal{B}\left(k_{o}\right)$ given in equation (12), the components of $G(k ; \beta)^{a b}$ read

$$
\begin{aligned}
G(k ; \beta)^{11} & =\frac{1}{k^{2}-m^{2}+\mathrm{i} \epsilon}-2 \pi \mathrm{i} n\left(k_{o}\right) \delta\left(k^{2}-m^{2}\right) \\
G(k ; \beta)^{22} & =\frac{-1}{k^{2}-m^{2}-\mathrm{i} \epsilon}-2 \pi \mathrm{i} n\left(k_{o}\right) \delta\left(k^{2}-m^{2}\right) \\
G(k ; \beta)^{12} & =G(k ; \beta)^{21}=-2 \pi \mathrm{i}\left[n\left(k_{o}\right)+n\left(k_{o}\right)^{2}\right]^{1 / 2} \delta\left(k^{2}-m^{2}\right) .
\end{aligned}
$$

At this point we can set forth rules for the construction of a general thermal theory. Let us denote by $\mathcal{L}_{T}=\{A, B, C, \ldots, \tilde{A}, \tilde{B}, \tilde{C} \ldots\}$ the set of dynamical variables in TFD defined in the Hilbert space $\mathcal{H}$ with elements $|\Phi\rangle=|\phi, \tilde{\phi}\rangle$. The action of generic operators $A$ and $\tilde{A}$ on $|\Phi\rangle$ is specified by

$$
\begin{aligned}
& A|\Phi\rangle \equiv \mathrm{A} \otimes 1(|\phi\rangle \otimes\langle\phi|)=(\mathrm{A}|\phi\rangle) \otimes\langle\phi| \\
& \tilde{A}|\Phi\rangle=1 \otimes \mathrm{A}(|\phi\rangle \otimes\langle\phi|)=|\phi\rangle \otimes\langle\phi| \mathrm{A}^{\dagger}
\end{aligned}
$$

where here the operator $A$ is defined in the usual Hilbert space, $\mathfrak{H}$, with $|\phi\rangle \in \mathfrak{H}$. Therefore, the Hilbert space in TFD, $\mathcal{H}$, is given by

$$
\mathcal{H}=\mathfrak{H} \otimes \mathfrak{H}^{*} .
$$

Equations (14) and (15) induce a mapping $\sim \mathcal{L}_{T} \mapsto \mathcal{L}_{T}$, called tilde (or dual) conjugation rules fulfilling the following properties:

$$
\begin{aligned}
& (A B)^{\tilde{A}}=\tilde{A} \tilde{B} \\
& (A+\alpha B)^{\tilde{A}}=\tilde{A}+\alpha^{*} \tilde{B} \\
& \left(A^{\dagger}\right)^{\tilde{A}}=(\tilde{A})^{\dagger} \\
& \tilde{\tilde{A}}=A \\
& {[A, \tilde{B}]=0 .}
\end{aligned}
$$

Beyond that, the space $\mathcal{H}$ can be used as a representation space for Lie algebras. To achieve this goal, let $\mathfrak{g}$ be a Lie algebra specified by $g_{i} \diamond g_{j}=C_{i j}^{k} g_{k}$, where $\diamond$ is the Lie product and $C_{i j}^{k}$ are the structure constants (we are assuming the rule of sum over repeated indices). A unitary representation of $\mathfrak{g}$ in $\mathcal{H}$ is then given by

$$
\begin{aligned}
& {\left[A_{i}, A_{j}\right]=\mathrm{i} C_{i j}^{k} A_{k}} \\
& {\left[\tilde{A}_{i}, \tilde{A}_{j}\right]=-\mathrm{i} C_{i j}^{k} \tilde{A}_{k}} \\
& {\left[A_{i}, \tilde{A}_{j}\right]=0 .}
\end{aligned}
$$

For proposals of physical interpretation, this algebra can be rewritten by the introduction of a (hat-) mapping, say ${ }^{\wedge}: \mathcal{L}_{T} \mapsto \mathcal{L}_{T}$, defined by $\hat{A}=A-\tilde{A}$, resulting from equations (17)-(19) in

$$
\begin{aligned}
& {\left[A_{i}, A_{j}\right]=C_{i j}^{k} A_{k}} \\
& {\left[\hat{A}_{i}, A_{j}\right]=C_{i j}^{k} A_{k}} \\
& {\left[\hat{A}_{i}, \hat{A}_{j}\right]=C_{i j}^{k} \hat{A}_{k} .}
\end{aligned}
$$


Note that this Lie algebra, to be denoted by $\mathfrak{g}_{T}$, as a vector space is given by $\mathfrak{g}_{T}=\mathfrak{g} \oplus \hat{\mathfrak{g}}$, where $\mathfrak{g}(\hat{\mathfrak{g}})$ is a sub-vector space of $\mathfrak{g}_{T}$ given by the non-hat (hat) operators.

Some aspects of $\mathfrak{g}_{T}$ have been studied in connection with relativistic kinetic theories $[6,12]$. Here, however, we explore representations of the normal group given by the invariant subgroup defined by the non-hat operators, that is, we study the normal algebra $\mathfrak{n}(\mathfrak{g})=\mathfrak{g}_{T} / \mathfrak{g}$ in the case of Poincaré symmetries. Then we show how to build up the (classical and quantum) relativistic kinetic theory in a unified way using the notion of Lie group representation.

\section{Representations of the Poincaré group in $\mathcal{H}$}

The $\mathfrak{g}_{T}$ Poincaré-Lie algebra, which will be denoted by $\mathfrak{p}_{T}$, is given by [12]

$$
\begin{aligned}
& {\left[M_{\mu \nu}, P_{\sigma}\right]=\mathrm{i}\left(g_{\nu \sigma} P_{\mu}-g_{\sigma \mu} P_{v}\right)} \\
& {\left[P_{\mu}, P_{\nu}\right]=0} \\
& {\left[M_{\mu \nu}, M_{\sigma \rho}\right]=-\mathrm{i}\left(g_{\mu \rho} M_{\nu \sigma}-g_{\nu \rho} M_{\mu \sigma}+g_{\mu \sigma} M_{\rho v}-g_{\nu \sigma} M_{\rho \mu}\right)} \\
& {\left[\hat{M}_{\mu \nu}, P_{\sigma}\right]=\left[M_{\mu \nu}, \hat{P}_{\sigma}\right]=\mathrm{i}\left(g_{\nu \sigma} P_{\mu}-g_{\sigma \mu} P_{\nu}\right)} \\
& {\left[\hat{P}_{\mu}, P_{\nu}\right]=0} \\
& {\left[\hat{M}_{\mu \nu}, M_{\sigma \rho}\right]=-\mathrm{i}\left(g_{\mu \rho} M_{\nu \sigma}-g_{\nu \rho} M_{\mu \sigma}+g_{\mu \sigma} M_{\rho \nu}-g_{\nu \sigma} M_{\rho \mu}\right)} \\
& {\left[\hat{M}_{\mu \nu}, \hat{P}_{\sigma}\right]=\mathrm{i}\left(g_{\nu \sigma} \hat{P}_{\mu}-g_{\sigma \mu} \hat{P}_{\nu}\right)} \\
& {\left[\hat{P}_{\mu}, \hat{P}_{\nu}\right]=0} \\
& {\left[\hat{M}_{\mu \nu}, \hat{M}_{\sigma \rho}\right]=-\mathrm{i}\left(g_{\mu \rho} \hat{M}_{\nu \sigma}-g_{\nu \rho} \hat{M}_{\mu \sigma}+g_{\mu \sigma} \hat{M}_{\rho \nu}-g_{\nu \sigma} \hat{M}_{\rho \mu}\right)}
\end{aligned}
$$

where the metric tensor is such that $\operatorname{diag}\left(g_{\mu \nu}\right)=(1,-1,-1,-1)$, and $g_{\mu \nu}=0$ for $\mu \neq v$ where $\mu, v=0,1,2,3$. As for the general case of $\mathfrak{g}_{T}$, as a vector space $\mathfrak{p}_{T}=\mathfrak{p} \oplus \hat{\mathfrak{p}}$, where $\mathfrak{p}(\hat{\mathfrak{p}})$ is a sub-vector space of $\mathfrak{p}_{T}$ given by the non-hat (hat) operators. Furthermore, using standard methods of group theory [22], the normal algebra $\mathfrak{n}(\mathfrak{p})=\mathfrak{p}_{T} / \mathfrak{p}$ is given by

$$
\begin{aligned}
& {\left[\hat{M}_{\mu \nu}, \hat{M}_{\sigma \rho}\right]=\mathrm{i}\left(g_{\mu \sigma} \hat{M}_{\rho \nu}+g_{\rho \nu} \hat{M}_{\sigma \mu}+g_{\mu \rho} \hat{M}_{\nu \sigma}+g_{\sigma \nu} \hat{M}_{\mu \rho}\right)} \\
& {\left[\hat{M}_{\mu \nu}, \hat{P}_{\rho}\right]=\mathrm{i}\left(g_{\mu \rho} \hat{P}_{\nu}-g_{\nu \rho} \hat{P}_{\mu}\right)} \\
& {\left[\hat{M}_{\mu \nu}, M_{\sigma \rho}\right]=\mathrm{i}\left(g_{\mu \sigma} M_{\rho \nu}+g_{\rho \nu} M_{\sigma \mu}+g_{\mu \rho} M_{\nu \sigma}+g_{\sigma \nu} M_{\mu \rho}\right)} \\
& {\left[\hat{M}_{\mu \nu}, P_{\sigma}\right]=\left[M_{\mu \nu}, \hat{P}_{\sigma}\right]=\mathrm{i}\left(g_{\mu \sigma} P_{\nu}-g_{\nu \sigma} P_{\mu}\right)}
\end{aligned}
$$

all the other commutation relations are null. A similar kind of Lie algebra was studied, for instance, by Gulmanelli [23], but was fully based on the adjoint representation of classical mechanics. This is not the case here where we are interested in general unitary representations.

Three invariants of $\mathfrak{n}(\mathfrak{p})$ are immediately identified as

$$
\begin{aligned}
& I_{1}=P^{\mu} P_{\mu} \\
& I_{2}=\hat{P}^{\mu} \hat{P}_{\mu} \\
& I_{3}=P^{\mu} \hat{P}_{\mu} .
\end{aligned}
$$

Since that the non-hat operators form an invariant Abelian algebra, we can take such operators and the invariants $I_{1}, I_{2}, I_{3}$ to build a Hilbert space frame. In order to do so, we first note that

$$
\begin{aligned}
& \mathrm{e}^{-\alpha_{\mu} \hat{P}^{\mu}} P_{\sigma} \mathrm{e}^{\alpha_{\nu} \hat{P}^{v}}=P_{\sigma} \\
& \mathrm{e}^{-\alpha_{\mu} \hat{P}^{\mu}} M_{\sigma \rho} \mathrm{e}^{\alpha_{\nu} \hat{P}^{v}}=M_{\sigma \rho}+\alpha_{\sigma} P_{\rho}-\alpha_{\rho} P_{\sigma} \\
& \mathrm{e}^{-\varepsilon_{\mu \nu} \hat{M}^{\mu \nu}} P_{\sigma} \mathrm{e}^{\varepsilon_{\mu \nu} \hat{M}^{\mu \nu}}=P_{\sigma}+\mathrm{i}\left(\varepsilon_{\sigma \nu} P^{v}-\varepsilon_{\nu \sigma} P^{v}\right) .
\end{aligned}
$$


Therefore, we can view $P_{\sigma}$ and $M_{\sigma \rho}$ as natural candidates for describing linear and angular momentum, respectively. On the other hand, $\hat{P}^{\mu}$ and $\hat{M}^{\mu v}$ can be taken as the generators of translations and rotations, respectively. That this interpretation is consistent can be verified, for instance, from equation (34) which establishes that under rotations the linear momentum is transformed as a 4-vector. This interpretative aspect is a direct consequence of the fact that the algebra of the non-hat operators is an invariant subalgebra of $\mathfrak{n}(\mathfrak{p})$. Now we assume the existence of a Hilbert space $\mathcal{H}(\mathfrak{n}(\mathfrak{p}))$ on which the elements of $\mathfrak{n}(\mathfrak{p})$ are defined and introduce a non-hat operator, say $Q_{\mu}$, through the condition

$$
\mathrm{e}^{-\alpha_{\mu} \hat{P}^{\mu}} Q_{\sigma} \mathrm{e}^{\alpha_{\nu} \hat{P}^{v}}=Q_{\sigma}+\mathrm{i} \alpha_{\sigma}
$$

where $\alpha_{\sigma}$ is a constant. So doing, the operator $Q_{\mu}$ describes positions. Therefore, a (phasespace) frame for the Hilbert space $\mathcal{H}(\mathfrak{n}(\mathfrak{p}))$ can be introduced since $[P, Q]=0$. Let us thus define $|q, p\rangle \in \mathcal{H}(\mathfrak{n}(\mathfrak{p}))$ such that $P|q, p\rangle=p|q, p\rangle, Q|q, p\rangle=q|q, p\rangle$, with $q$ and $p$ being real 4-vectors, and $\langle q, p \mid \phi\rangle=\phi(q, p)$ being an $L^{2}$ (Lebesgue)-type function, that is $\int|\phi(q, p)|^{2} \mathrm{~d} q \mathrm{~d} p<\infty$. This last condition is used to impose the normalization condition. In this way, we obtain a unitary representation for $\mathfrak{n}(\mathfrak{p})$ writing

$$
\begin{aligned}
& M_{\mu \nu}=P_{\mu} Q_{\nu}-P_{\nu} Q_{\mu} \\
& \hat{M}_{\mu \nu}=P_{\mu} \hat{Q}_{\nu}-P_{\nu} \hat{Q}_{\mu}+Q_{\nu} \hat{P}_{\mu}-Q_{\mu} \hat{P}_{\nu}
\end{aligned}
$$

where

$$
\hat{P}_{\mu}=-\mathrm{i} \frac{\partial}{\partial q_{\mu}} \quad \hat{Q}_{\mu}=\mathrm{i} \frac{\partial}{\partial p_{\mu}} \quad P_{\mu}=1 \cdot p_{\mu} \quad Q_{\mu}=1 \cdot q_{\mu} .
$$

A general association between a hat and a non-hat operator, consistent with equations (35)(37), is thus introduced. Considering an arbitrary function of the phase space, say $A(q, p)$, then we have two mappings, (i) c: $A(q, p) \rightarrow \mathrm{A}=1 \cdot A(q, p)$, giving rise to c-number operators, and (ii) ${ }^{\wedge}: A(q, p) \rightarrow \hat{A}$, such that $\hat{A}=(\hat{p} A(q, p)) \hat{q}+\alpha(\hat{q} A(q, p)) \hat{p}+\beta \hat{q}(\hat{p} A(q, p))$, where $\alpha$ and $\beta$ are constants. In the case in which $\alpha=1$ and $\beta=0$ the association is such that $A \rightarrow \hat{A}=\mathrm{i}\{A(q, p), \cdot\}$, where $\{\cdot, \cdot\}$ is the Poisson bracket. In this situation, we have

$$
(\gamma A+B)^{\wedge}=\gamma(\hat{A}+\hat{B}) \quad(A B)^{\wedge}=A \hat{B}+\hat{B} A \quad(\gamma)^{\hat{n}}=0
$$

where $\gamma$ is a constant. This result is useful to derive another set of Casimir invariants of $\mathfrak{n}(\mathfrak{p})$. Defining $w_{u}=\frac{1}{2} \varepsilon_{\mu \nu \sigma \rho} M^{\nu \sigma} P^{\rho}$, the Pauli-Lubanski vector, we find the following invariants: $W=w^{\mu} w_{u}$ and $\hat{W}=2 w^{\mu} \hat{w}_{u}$. Hence, we can write equations (35) and (36) respectively as: $M_{\mu \nu} \rightarrow J_{\mu \nu}=M_{\mu \nu}+S_{\mu \nu}$ and $\hat{M}_{\mu \nu} \rightarrow \hat{J}_{\mu \nu}=\hat{M}_{\mu \nu}+\hat{S}_{\mu \nu}$, where the variables $S_{\mu \nu}$ and $\hat{S}_{\mu \nu}$ are related to the spinorial index of the representation, which will be taken here as zero (scalar representations).

We assume that the average of a one-body diagonal operator is given by

$$
\begin{aligned}
\langle\bar{A}\rangle & =\langle\phi|\bar{A}| \phi\rangle \\
& =\int \mathrm{d} q \mathrm{~d} p \mathrm{~d} q^{\prime} \mathrm{d} p^{\prime}\langle\phi \mid q, p\rangle\left\langle q, p|\bar{A}| q^{\prime}, p^{\prime}\right\rangle\left\langle q^{\prime}, p^{\prime} \mid \phi\right\rangle \\
& =\int \mathrm{d} q \mathrm{~d} p \mathrm{~d} q^{\prime} \mathrm{d} p^{\prime} \phi^{*}(q, p) \bar{A}\left(q, p, q^{\prime}, p^{\prime}\right) \delta^{4}\left(q-q^{\prime}\right) \delta^{4}\left(p-p^{\prime}\right) \phi\left(q^{\prime}, p^{\prime}\right) \\
& =\int \mathrm{d} q \mathrm{~d} p \phi^{*}(q, p) \bar{A}(q, p) \phi(q, p)
\end{aligned}
$$

where the notation $\bar{A}$ stands for either a c-number operator, $A$, or a hat operator, $\hat{A}$. If $\bar{A}=A$, then the average of $A$ reduces to $\langle A\rangle=\int \mathrm{d} q \mathrm{~d} p f(q, p) A(q, p)$, where $f(q, p)=|\phi(q, p)|^{2}$. 
Let us now write down an equation of motion for $\phi(q, p)$. According to the Schur's lemma [22], the invariant $I_{3}$, given in equation (31), has a fixed value in this phase-space representation. Then, considering $I_{3}=0$, from equation (37) we write

$$
p^{\mu} \frac{\partial}{\partial q^{\mu}} \phi(q, p)=0
$$

which is a collisionless transport equation, also furnished by the positive-defined real quantity $f(q, p)$. Then we can interpret $\phi(q, p)$ as a probability amplitude in phase space and $f(q, p)$ as a (classical) probability density.

In order to complete the physical interpretation of this formalism, as well as for practical considerations, let us define the tensor

$$
\bar{T}^{\mu \nu}(q)=\frac{1}{2(2 \pi)^{3}} \int \frac{\mathrm{d}^{3} p}{p^{o}} \phi^{*}(q, p) \bar{P}^{\mu} \bar{P}^{v} \phi(q, p) .
$$

Taking, in particular, $\bar{P} \equiv P$, we arrive at the usual definition of the energy-momentum tensor $\bar{T}^{\mu \nu} \rightarrow T^{\mu \nu}(q)=\frac{1}{2(2 \pi)^{3}} \int \frac{\mathrm{d}^{3} p}{p^{o}} p^{\mu} p^{\nu} f(q, p)$, where $T^{00}(q)$ is the average value of the energy per particle, $T^{0 i}(q)$ is the average value of the energy flow, $T^{i 0}(q)$ is the macroscopic momentum flow and $T^{i j}(q)$ is the pressure tensor. This result shows the compatibility of our approach with the usual kinetic theory [24].

\section{Boltzmann equation and nonrelativistic limit}

In order to derive a collision term in the rhs of equation (39), we can use the notion of a propagator in the Hilbert phase space $\mathcal{H}(\mathfrak{n}(\mathfrak{p}))$. Consider the (pointwise) collision between two particles (1) and (2) with initial (i) and final (f) momenta specified, respectively, by $p_{i 1}^{\mu}, p_{i 2}^{\mu}$, $p_{f 1}^{\mu}$, and $p_{f 2}^{\mu}$. Now define a transition amplitude at a point $q$, say the propagator $W\left(q, p_{f 1}, p_{f 2}\right.$, $\left.p_{i 1} p_{i 2}\right)$ of the system, from a initial state $\left|q, p_{i 1} ; q, p_{i 2}\right\rangle$ to a final state $\left|q, p_{f 1} ; q, p_{f 2}\right\rangle$, that is $W\left(q, p_{f 1}, p_{f 2} \mid p_{i 1}, p_{i 2}\right)=\left\langle q, p_{f 1} ; q, p_{f 2} \mid q, p_{i 1} ; q, p_{i 2}\right\rangle$. Then, the transition amplitude for any point $q$ of space-time is

$$
W\left(p_{f 1}, p_{f 2} \mid p_{i 1}, p_{i 2}\right)=\int \mathrm{d}^{4} q\left\langle q, p_{f 1} ; q, p_{f 2} \mid q, p_{i 1} ; q, p_{i 2}\right\rangle
$$

such that we can write $\left\langle q, p_{i 1} ; q, p_{i 2}\right|=\int \mathrm{d}^{4} p_{f 1} \mathrm{~d}^{4} p_{f 2} W\left(p_{i 1}, p_{i 2} \mid p_{f 1}, p_{f 2}\right)\left\langle q, p_{f 1} ; q, p_{f 2}\right|$, and so we have

$$
\left\langle q, p_{i 1} ; q, p_{i 2} \mid \phi_{12}\right\rangle=\int \mathrm{d}^{4} p_{f 1} \mathrm{~d}^{4} p_{f 2} W\left(p_{i 1}, p_{i 2} \mid p_{f 1}, p_{f 2}\right)\left\langle q, p_{f 1} ; q, p_{f 2} \mid \phi_{12}\right\rangle .
$$

Using the indistinguishability of the particles, an intrinsic ingredient in our formalism since we are considering amplitudes, and the Hartree approximation $\left\langle q, p_{f 1} ; q, p_{f 2} \mid \phi_{12}\right\rangle=$ $\phi\left(q, p_{f 1}\right) \phi\left(q, p_{f 2}\right)$, we can compute the change in the probability amplitude, say $\Psi_{+}\left(q, p_{i 1}\right)$, due to particles leaving the collision at $q$ with momentum $p_{i 1}$, that is

$$
\Psi_{+}\left(q, p_{i 1}\right)=\frac{1}{2} \int \mathrm{d}^{4} p_{i 2} \mathrm{~d}^{4} p_{f 1} \mathrm{~d}^{4} p_{f 2} W\left(p_{f 1}, p_{f 2} \mid p_{i 1}, p_{i 2}\right) \phi\left(q, p_{f 1}\right) \phi\left(q, p_{f 2}\right) .
$$

The same reasoning can be used to compute the effect of particles leaving the collision at $q$ with momentum other than $p_{i 1}$, that is

$\Psi_{-}\left(q, p_{i 1}\right)=\frac{1}{2} \int \mathrm{d}^{4} p_{i 2} \mathrm{~d}^{4} p_{f 1} \mathrm{~d}^{4} p_{f 2} W\left(p_{i 1}, p_{i 2} \mid p_{f 1}, p_{f 2}\right) \phi\left(q, p_{f 1}\right) \phi\left(q, p_{f 2}\right)$.

Therefore, we obtain the following transport equation:

$$
p^{\mu} \partial_{\mu} \phi(q, p)=\mathcal{C}(q, p)
$$


where

$$
\begin{aligned}
\mathcal{C}(q, p)=\Psi_{+} & (q, p)-\Psi_{-}(q, p) \\
= & \frac{1}{2} \int \mathrm{d}^{4} p_{i 2} \mathrm{~d}^{4} p_{f 1} \mathrm{~d}^{4} p_{f 2}\left[W\left(p_{f 1}, p_{f 2} \mid p, p_{i 2}\right) \phi\left(q, p_{f 1}\right) \phi\left(q, p_{f 2}\right)\right. \\
& \left.-W\left(p, p_{i 2} \mid p_{f 1}, p_{f 2}\right) \phi\left(q, p_{f 1}\right) \phi\left(q, p_{f 2}\right)\right] .
\end{aligned}
$$

In consequence, we have derived a relativistic Boltzmann equation but rather for the probability amplitudes in phase space. An alternative method to the above is to derive the Boltzmann equation for the density, $f(q, p)$. This can be accomplished via the following substitutions in equation (40): $\phi(q, p) \rightarrow f(q, p)$ and $W\left(p_{f 1}, p_{f 2} \mid p, p_{i 2}\right) \rightarrow$ $\left|W\left(p_{f 1}, p_{f 2} \mid p, p_{i 2}\right)\right|^{2}$, such that now $\Psi_{+}\left(q, p_{i 1}\right)\left(\Psi_{-}\left(q, p_{i 1}\right)\right)$ is the increase (decrease) in the probability density due to particles leaving a collision at $q$ with momentum (other than) $p_{i 1}$. Nevertheless, equation (40), written in terms of amplitude, plays a role when interference of states is manifested. This aspect will be explored in a future work.

It should be emphasized that the formalism developed here is a generalization of the classical non-relativistic TFD which has been identified in [25]. In order to see this, we can analyse the non-relativistic limit of the $\mathfrak{n}(\mathfrak{p})$. Considering equations (35) and (36), we have that the components of the Lorentz boost generators can be written as

$$
\hat{L}_{0 m}=\frac{\hat{K}_{m}}{c}=\mathrm{i}\left(\frac{H}{c^{2}} \frac{\partial}{\partial p_{m}}-p_{m} \frac{\partial}{\partial H}+t \frac{\partial}{\partial q_{m}}-\frac{q_{m}}{c^{2}} \frac{\partial}{\partial t}\right) .
$$

Therefore, proceeding the contraction $c \rightarrow \infty$, we have $\hat{K}_{m}=\mathrm{i}\left(m \frac{\partial}{\partial p_{m}}+t \frac{\partial}{\partial q_{m}}\right)$, where $\hat{K}_{m}$ is the generator of the usual Galilean boost transformation. On the other hand, for the energy we have, $\lim _{c \rightarrow \infty} \frac{H}{c^{2}}=\lim _{c \rightarrow \infty} \frac{1}{c^{2}} \sqrt{m^{2} c^{2}+p_{j} p^{j}} \cong m, j=1,2,3$. The remaining components of the total angular momentum operator also reduce to the Galilean counterpart. In addition, with the above representation for the total energy operator, in the non-relativistic limit the time-translation generator $\hat{P}_{o}$ becomes $\hat{H}=\mathrm{i} \partial_{t}$. Such operators are simply the ones used in [25]. Also observe that as in [25], we can introduce a Fock space representation via $\mathcal{H}(\mathfrak{n}(\mathfrak{p})) \otimes \mathcal{H}(\mathfrak{n}(\mathfrak{p})) \otimes \cdots \otimes \mathcal{H}(\mathfrak{n}(\mathfrak{p}))$, such that the amplitude $\phi(q, p)$ can be taken as field operators in phase space.

\section{Quantum formalism}

At this point, we would like to deal with the issue of quantization of such a classical formalism. As a first attempt, we can contemplate using a relativistic generalization of van Hove's quantization scheme [26], that is, let us define

$\hat{A}=A(q, p)-\frac{1}{2}\left(q_{\mu} \frac{\partial A(q, p)}{\partial q_{\mu}}+p_{\mu} \frac{\partial A(q, p)}{\partial p_{\mu}}\right)+\mathrm{i}\left(\frac{\partial A(q, p)}{\partial q^{\mu}} \frac{\partial}{\partial p_{\mu}}-\frac{\partial A(q, p)}{\partial p^{\mu}} \frac{\partial}{\partial q_{\mu}}\right)$.

In this case, the association of the hat and non-hat operators is given by

$$
(\alpha A+B) \hat{)}=\alpha(\hat{A}+\hat{B}) \quad(A B) \hat{)}=A \hat{B}+\hat{B} A-A B \quad(\alpha)^{\hat{}}=\alpha .
$$

Introducing, from equation (41), the set of operators

$$
\begin{aligned}
& Q=1 \cdot q \quad P=1 \cdot p \quad \hat{Q}=q / 2+\mathrm{i} \partial / \partial p \quad \hat{P}=p / 2-\mathrm{i} \partial / \partial q \\
& \text { we define } \\
& M_{\mu \nu}=P_{\mu} Q_{\nu}-P_{\nu} Q_{\mu} \\
& \hat{M}_{\mu \nu}=P_{\mu} \hat{Q}_{\nu}-P_{\nu} \hat{Q}_{\mu}+Q_{\nu} \hat{P}_{\mu}-Q_{\mu} \hat{P}_{\nu}+P_{\nu} Q_{\mu}-P_{\mu} Q_{\nu} .
\end{aligned}
$$


In addition, as we can verify by direct calculation, these operators provide another representation for the algebra $\mathfrak{n}(\mathfrak{p})$. This fact indicates a quantum theory. There is, however, a connection between the operators given in equation (37) and the correspondent ones defined by equation (43). Indeed,

$$
\left(\begin{array}{c}
Q \\
P \\
\hat{Q} \\
\hat{P}
\end{array}\right) \stackrel{U}{\rightarrow}\left(\begin{array}{c}
Q \\
P \\
\hat{Q} \\
\hat{P}
\end{array}\right)=\left(\begin{array}{cccc}
1 & 0 & 0 & 0 \\
0 & 1 & 0 & 0 \\
\frac{1}{2} & 0 & \mathrm{i} & 0 \\
0 & \frac{1}{2} & 0 & \mathrm{i}
\end{array}\right)\left(\begin{array}{c}
Q \\
P \\
\hat{Q} \\
\hat{P}
\end{array}\right)
$$

This result suggests that we can try to build a quantum formalism by a proper physical reinterpretation of the representations for $\mathfrak{n}(\mathfrak{p})$. In this way, note first that the meaning of $\phi(q, p)=\langle q p \mid \phi\rangle$ as a classical amplitude in phase was possible due to our interpretation of $Q$ and $P$, which commute with each other, as the position and momentum observables, respectively. In the sense of a quantum theory, looking for non-commutant observables, we have, however, other possibilities for the momentum and position operators. For instance, in equation (37), $Q$ and $\hat{P}$ transform as position and momentum, but now these operators do not commute with each other, that is $\left[Q_{\mu}, \hat{P}_{\nu},\right]=\mathrm{i} g_{\mu \nu}$. Despite the interpretation and because of the transformation properties, the operators $Q$ and $P$ can always be taken to define a phasespace basis of the Hilbert space. As a consequence, we can conjecture that equation (39) can be interpreted as a kind of phase-space Liouville-von Neuman equation for the scalar field. The physical consistency of this interpretation can be verified if we establish a connection of $\phi(q, p)$ with a density matrix and a Wigner density for the Klein-Gordon field. Let us then proceed in this direction, multiplying equation (39) by $\exp (\mathrm{i} p \cdot s)$ and integrating in $p$. Then equation (39) reads $\partial_{q} \partial_{s} \psi(q, s)=0$, where $\psi(q, s)=\int \mathrm{d}^{4} p \phi(q, p) \exp (\mathrm{i} p \cdot s)$. Now defining $x^{\prime}=\frac{1}{\sqrt{2}}(q+s)$ and $x=\frac{1}{\sqrt{2}}(q-s)$, such that $\psi(q, s)=\psi\left(x^{\prime}+x, x^{\prime}-x\right) \equiv \psi\left(x, x^{\prime}\right)$, we obtain

$$
\left(\partial_{x^{\prime}} \partial_{x^{\prime}}-\partial_{x} \partial_{x}\right) \psi\left(x, x^{\prime}\right)=0 .
$$

Writing $\psi\left(x, x^{\prime}\right)=\langle x \mid \psi\rangle \otimes\left\langle\psi \mid x^{\prime}\right\rangle$, from equation (47) we have a Liouville-von Neumann equation for the Klein-Gordon field, that is $(\square \otimes 1-1 \otimes \square)|\psi\rangle \otimes\langle\psi|=0$. Indeed, multiplying the rhs (lhs) of this equation by $|\psi\rangle$ (by $\langle\psi|$ ), and using the fact that $\langle\psi|\square| \psi\rangle=m^{2}$, we derive the Klein-Gordon equation $\left(\square-m^{2}\right)|\psi\rangle=0\left(\langle\psi|\left(\square-m^{2}\right)=0\right)$.

The connection of $\phi(q, p)$ with a Wigner density, $f_{W}(x, p)$, is therefore obtained via $\psi(q, s)=\psi^{*}\left(x+x^{\prime}\right) \psi\left(x-x^{\prime}\right)$; that is, since by definition, $f_{W}(x, p)=\int \mathrm{d}^{4} x^{\prime} \psi^{*}(x+$ $\left.x^{\prime}\right) \psi\left(x-x^{\prime}\right) \exp \left(-\mathrm{i} p \cdot x^{\prime}\right)$, then

$f_{W}(x, p)=\int \mathrm{d}^{4} x^{\prime} \mathrm{d}^{4} p^{\prime} \phi\left(x+x^{\prime}, p^{\prime}\right) \exp \left(\mathrm{i}\left(\frac{1}{\sqrt{2}} p^{\prime}+p\right) x^{\prime}\right) \operatorname{expi}\left(\frac{1}{\sqrt{2}} p^{\prime} x\right)$.

Therefore, the method developed here provides a scheme to derive the Wigner density directly from group theory. It is worthwhile noting that following the TFD construction presented in section 2 (and also in Bohm and Hiley [18] and Holland [19]), $\phi(q, p)$ in equation (39) can in turn be interpreted as a kind of square root of the Wigner function. In our context this means that we consider $|\psi\rangle \otimes\langle\psi|$ as a vector in the TFD Hilbert space, such that according to equation (6), $|\psi\rangle \otimes\langle\psi|\rightarrow| \psi\rangle \otimes|\tilde{\psi}\rangle=|\psi, \tilde{\psi}\rangle=\rho^{1 / 2}|1\rangle$.

\section{Concluding remarks}

Summarizing, in this work we have analysed representations of the Poincare group taking as the representation space the Hilbert space introduced in thermofield dynamics. From algebraic ingredients, we could recognize representations giving rise to classical kinetic theory 
in relativistic phase space, but based on the wavefunction notion. This was used to show how it is possible to derive a Boltzmann equation but using the notion of a propagator. Therefore, to relativistic situations we have generalized the Galilean classical approaches based on phasespace wavefunctions and second-quantization methods.

In addition, we have shown that, for classical systems, a class of operators in $\mathfrak{n}(\mathfrak{p})$ must be considered only as generators of transformation symmetries (as is the case of the operator $\hat{P}$ in equation (37)), while in a quantum approach in phase space, such a class is supposed to be interpreted but in association to observables. Moreover, equation (39) is a van Hove flow, since the invariant $p^{\mu} \partial_{\mu}$ is written in terms of a linear combination the operators $P$ and $\hat{P}$ given in equation (43), that is $p^{\mu} \partial_{\mu}=P^{2} / 2-P \hat{P}$. The association of $\phi(q, p)$ with the Wigner function is given in equation (48).

\section{Acknowledgments}

The authors thank M Revzen for the discussions and suggestions, and CAPES and CNPq (two Brazilian Government Agencies) for financial support.

\section{References}

[1] Fetter A L and Walecka J D 1971 Quantum Theory of Many-Particles Systems (New York: McGraw-Hill)

[2] Takahashi Y and Umezawa H 1975 Collect. Phenom. 255 Reprinted as Takahashi Y and Umezawa H 1996 Int. J. Mod. Phys. 101755

[3] Umezawa H 1993 Advanced Field Theory: Micro, Macro and Thermal Physics (New York: AIP)

[4] Le Bellac M 1996 Thermal Field Theory (Cambridge: Cambridge University Press)

[5] Ojima I 1981 Ann. Phys., NY 1371

[6] Santana A E, Matos Neto A, Vianna J D M and Khanna F C 1999 Int. J. Theor. Phys. 38641

[7] Mann A and Revzen M 1989 Phys. Lett. A 134273

[8] Mann A, Revzen M, Umezawa H and Yamanaka Y 1989 Phys. Lett. A 140475

[9] Kopf T, Santana A E and Khanna F C 1997 J. Math. Phys. 384971

[10] Celeghini E et al 1998 Phys. Lett. A 244455

[11] Whitehead J P, Matsumotov H and Umezawa H 1984 Phys. Lett. A 103408

[12] Santana A E and Khanna F C 1995 Phys. Lett. A 20368

[13] Zhuang P and Heinz U 1996 Ann. Phys., NY 245311

[14] Nayak G C and Ravshankar V 1997 Phys. Rev. D 556877

[15] Schönberg M 1952 Nuovo Cimento 121139

[16] Matos Neto A and Vianna J D M 1985 Nuovo Cimento B 86117

[17] Mattis D C and Glasser M L 1998 Rev. Mod. Phys. 70979

[18] Bohm D and Hiley B J 1983 Old and New Questions in Physics, Cosmology, Philosophy and Theoretical Biology ed A van der Merwe (New York: Plenum) p 67

[19] Holland P R 1986 Found. Phys. 16701

[20] Arimitsu T and Umezawa H 1985 Prog. Theor. Phys. 74429

[21] Henning P A, Becker Ch, Lang A and Winkler U 1989 Phys. Lett. B 217211

[22] Sternberg S 1994 Group Theory and Physics (Cambridge: Cambridge University Press)

[23] Gulmanelli G 1963 Phys. Lett. 5320

[24] De Groot S R, Van Leeuwen W A and Van Weert Ch G 1980 Relativistic Kinetic Theory-Principles and Applications (New York: North-Holland)

[25] Santana A E, Khanna F C, Chu H and Chang Y C 1996 Ann. Phys., NY 246481

[26] Van Hove L 1951 Proc. R. Acad. Sci. 261

See also

Papaloucas L C and Rembielinski J 1989 J. Phys. A: Math. Gen. 222751

Woodhouse N M J 1992 Geometrical Quantization (Oxford: Clarendon) 